H.-X. YI

KODAI MATH. J.

13 (1990), 39-46

\title{
A QUESTION OF C. C. YANG ON THE UNIQUENESS OF ENTIRE FUNCTIONS
}

\author{
BY HONG-XUN YI
}

\section{Introduction and Main Results}

Let $f$ and $g$ be two nonconstant entire functions. If $f$ and $g$ have the same $a$-points with the same multiplicities, we denote this by $f=a \rightleftarrows g=a$ for simplicity's sake (see, [1]). It is assumed that the reader is familiar with the notations of the Nevanlinna Theory (see, for example, [2]). We denote by $S(r, f)$ any quantity satisfying $S(r, f)=o(T(r, f))$ as $r \rightarrow \infty$ except possibly for a set of $r$ of finite linear measure.

M. Ozawa has proved the following theorem:

THEOREM A (see [1]). Let $f$ and $g$ be entire functions of finite order. Assume that $f=0 \rightleftarrows g=0, f=1 \rightleftarrows g=1$ and $\delta(0, f)>1 / 2$. Then $f \cdot g \equiv 1$ unless $f \equiv g$.

In [3] H. Ueda has shown that in Theorem A the order restriction of $f$ and $g$ can be removed. He proved the following theorem:

Theorem B. Let $f$ and $g$ be entire functions. Assume that $f=0 \rightleftarrows g=0$, $f=1 \rightleftarrows g=1$ and $\delta(0, f)>1 / 2$. Then $f \cdot g \equiv 1$ unless $f \equiv g$.

In [4] C. C. Yang has asked: what can be said about the relationship between two entire functions $f$ and $g$ if $f=0 \rightleftarrows g=0$ and $f^{\prime}=1 \rightleftarrows g^{\prime}=1$ ?

In this paper we answer the question posed by C.C. Yang. In fact, we prove the following theorem:

THEOREM 1. Let $f$ and $g$ be two nonconstant entire functions. Assume that $f=0 \rightleftarrows g=0, f^{\prime}=1 \rightleftarrows g^{\prime}=1$ and $\delta(0, f)>1 / 2$. Then $f^{\prime} g^{\prime} \equiv 1$ unless $f \equiv g$.

The assumption " $\delta(0, f)>1 / 2$ " in Theorem 1 is best possible. Indeed, consider

$$
f(z)=-\frac{1}{2} e^{2 z}-\frac{1}{2} e^{z}, \quad g(z)=\frac{1}{2} e^{-2 z}+\frac{1}{2} e^{-z} .
$$

Then $f=0 \rightleftarrows g=0, f^{\prime}=1 \rightleftarrows g^{\prime}=1$ and $\delta(0, f)=1 / 2 . \quad f \not \equiv g$ and $f^{\prime} \cdot g^{\prime} \not \equiv 1$ are evident.

In place of Theorem 1, we prove more generally the following theorem 
which includes Theorem B and Theorem 1.

TheORem 2. Let $f$ and $g$ be two nonconstant entire functions. Assume that $f=0 \rightleftarrows g=0, f^{(n)}=1 \rightleftarrows g^{(n)}=1$ and $\delta(0, f)>1 / 2$, where $n$ is a nonnegative integer. Then $f^{(n)} \cdot g^{(n)} \equiv 1$ unless $f \equiv g$.

Theorem 2 is the best possible. Indeed, let

$$
\begin{aligned}
& f(z)=-\frac{1}{2^{n}} e^{2 z}+\frac{(-1)^{n+1}}{2^{n}} e^{z}, \\
& g(z)=\frac{(-1)^{n+1}}{2^{n}} e^{-2 z}-\frac{1}{2^{n}} e^{-z},
\end{aligned}
$$

where $n$ is a non-negative integer. It is easy to see that $f=0 \rightleftarrows g=0, f^{(n)}=1 \rightleftarrows$ $g^{(n)}=1$ and $\delta(0, f)=1 / 2$, but $f \not \equiv g$ and $f^{(n)} \cdot g^{(n)} \not \equiv 1$. This shows that $\delta(0, f)$ $>1 / 2$ is needed.

\section{Some Lemmas}

The following Lemmas will be needed in the proof of our theorems.

LEMMA 1 (see [2]). Let $f$ be a nonconstant entire function, $n$ be a nonnegative integer. Then

$$
T\left(r, f^{(n)}\right) \leqq T(r, f)+S(r, f) .
$$

LEMMA 2. Under the same conditions of Lemma 1, we have

$$
N\left(r, \frac{1}{f^{(n)}}\right) \leqq T\left(r, f^{(n)}\right)-T(r, f)+N\left(r, \frac{1}{f}\right)+S(r, f) .
$$

Proof. We note that

$$
\begin{aligned}
m\left(r, \frac{1}{f}\right) & \leqq m\left(r, \frac{1}{f^{(n)}}\right)+m\left(r, \frac{f^{(n)}}{f}\right) \\
& =m\left(r, \frac{1}{f^{(n)}}\right)+S(r, f) .
\end{aligned}
$$

By the first fundamental theorem (see [2]). we have from (1),

$$
T(r, f)-N\left(r, \frac{1}{f}\right) \leqq T\left(r, f^{(n)}\right)-N\left(r, \frac{1}{f^{(n)}}\right)+S(r, f) .
$$

Thus

$$
N\left(r, \frac{1}{f^{(n)}}\right) \leqq T\left(r, f^{(n)}\right)-T(r, f)+N\left(r, \frac{1}{f}\right)+S(r, f),
$$

which proves Lemma 2 . 
LEMMA 3. Let $g$ be a nonconstant entire function, $n$ be a nonnegative integer. Then

$$
N\left(r, \frac{1}{g^{(n)}}\right) \leqq N\left(r, \frac{1}{g}\right)+S(r, g) .
$$

Proof. By Lemma 2 we have

$$
N\left(r, \frac{1}{g^{(n)}}\right) \leqq T\left(r, g^{(n)}\right)-T(r, g)+N\left(r, \frac{1}{g}\right)+S(r, g) \text {. }
$$

From Lemma 1 we have

Hence

$$
T\left(r, g^{(n)}\right) \leqq T(r, g)+S(r, g) .
$$

$$
N\left(r, \frac{1}{g^{(n)}}\right) \leqq N\left(r, \frac{1}{g}\right)+S(r, g),
$$

which proves Lemma 3.

Lemma 4. Assume that the conditions of Theorem 2 are satisfied. Then

$$
\begin{array}{ll}
T(r, f)=O\left(T\left(r, f^{(n)}\right)\right) & r(\notin E), \\
T(r, g)=O\left(T\left(r, f^{(n)}\right)\right. & (r \notin E),
\end{array}
$$

where $E$ is a set of finite linear measure.

Proof. From (1) we get

$$
(\delta(0, f)+o(1)) T(r, f) \leqq T\left(r, f^{(n)}\right)+S(r, f) .
$$

Hence we have

$$
T(r, f) \leqq\left(\frac{1}{\delta(0, f)}+o(1)\right) T\left(r, f^{(n)}\right) \quad(r \notin E),
$$

that is

$$
T(r, f)=O\left(T\left(r, f^{(n)}\right)\right) \quad(r \notin E) .
$$

By Milloux's basic result (see, for example, [2, Theorem 3.2]), we have

$$
T(r, g)<N\left(r, \frac{1}{g}\right)+N\left(r, \frac{1}{g^{(n)}-1}\right)+S(r, g) \text {. }
$$

We note that

$$
\begin{aligned}
N\left(r, \frac{1}{g}\right) & =N\left(r, \frac{1}{f}\right) \leqq(1-\delta(0, f)+o(1)) T(r, f) \\
& \leqq(1-\delta(0, f)+o(1))\left(\frac{1}{\delta(0, f)}+o(1)\right) T\left(r, f^{(n)}\right) \\
& =\left(\frac{1}{\delta(0, f)}-1+o(1)\right) T\left(r, f^{(n)}\right) \quad(r \in E)
\end{aligned}
$$


and

$$
N\left(r, \frac{1}{g^{(n)}-1}\right)=N\left(r, \frac{1}{f^{(n)}-1}\right) \leqq T\left(r, f^{(n)}\right)+O(1) .
$$

From (6), (7), (8) we obtain

$$
T(r, g) \leqq\left(\frac{1}{\delta(0, f)}+o(1)\right) T\left(r, f^{(n)}\right)+S(r, g),
$$

that is

$$
T(r, g)=O\left(T\left(r, f^{(n)}\right)\right) \quad(r \notin E) .
$$

This completes the proof of Lemma 4 .

LEMMA 5. Let $f_{1}$ and $f_{2}$ be two nonconstant entire functions, and let $c_{1}, c_{2}$ and $c_{3}$ be three nonzero constants. If $c_{1} f_{1}+c_{2} f_{2} \equiv c_{3}$, then

$$
T\left(r, f_{1}\right)<N\left(r, \frac{1}{f_{1}}\right)+N\left(r, \frac{1}{f_{2}}\right)+S\left(r, f_{1}\right) .
$$

Proof. By the second fundamental theorem (see [2]), we have

$$
\begin{aligned}
T\left(r, f_{1}\right) & <N\left(r, \frac{1}{f_{1}}\right)+N\left(r, \frac{1}{f_{1}-\frac{c_{3}}{c_{1}}}\right)+S\left(r, f_{1}\right) \\
& =N\left(r, \frac{1}{f_{1}}\right)+N\left(r, \frac{1}{f_{2}}\right)+S\left(r, f_{1}\right),
\end{aligned}
$$

which proves Lemma 5.

Lemma 6 (see [5], [6]). Let $f_{1}, f_{2}, \cdots, f_{n}$ be linearly independent entire functions satisfying $\sum_{i=1}^{n} f_{i} \equiv 1$. Then for $j=1,2, \cdots, n$ we have

$$
T\left(r, f_{j}\right)<\sum_{i=1}^{n} N\left(r, \frac{1}{f_{i}}\right)+O(\log r+\log T(r)) \quad(r \notin E),
$$

where $T(r)$ denotes the maximum of $T\left(r, f_{\imath}\right), i=1,2, \cdots, n$.

This is a special case of a result of $\mathrm{R}$. Nevanlinna (see, $\left[5, \mathrm{P}_{116}\right]$ ).

To prove our theorems, we also need the following result, which is interesting by itself.

LEMMA 7. Let $f_{1}, f_{2}$ and $f_{3}$ be three entire functions satisfying

$$
\sum_{i=1}^{3} f_{i} \equiv 1 \text {. }
$$

If $f_{1} \not \equiv$ constant, and 


$$
\sum_{i=1}^{3} N\left(r, \frac{1}{f_{i}}\right) \leqq(\lambda+o(1)) T(r) \quad(r \notin E)
$$

where $T(r)=\max _{\imath=1,2,3}\left\{T\left(r, f_{\imath}\right)\right\}$, and $\lambda<1$, then $f_{2} \equiv 1$ or $f_{3} \equiv 1$.

Proof. Suppose neither $f_{2}$ nor $f_{3}$ are constants. If $f_{1}, f_{2}$ and $f_{3}$ are linearly independent, by Lemma 6 and (10) we have

and hence

$$
\begin{aligned}
T\left(r, f_{\jmath}\right) & <\sum_{i=1}^{3} N\left(r, \frac{1}{f_{i}}\right)+o(T(r)) \\
& \leqq(\lambda+o(1)) T(r) \quad(r \notin E, j=1,2,3)
\end{aligned}
$$

$$
T(r) \leqq(\lambda+o(1)) T(r) \quad(r \notin E)
$$

which is impossible. If $f_{1}, f_{2}$ and $f_{3}$ are linearly dependent, there exist three constants $\left(c_{1}, c_{2}, c_{3}\right) \neq(0,0,0)$ such that

$$
\sum_{i=1}^{3} c_{\imath} f_{i} \equiv 0
$$

Assume $c_{1} \neq 0$, from (9), (12) we have

$$
\left(1-\frac{c_{2}}{c_{1}}\right) f_{2}+\left(1-\frac{c_{3}}{c_{1}}\right) f_{3} \equiv 1
$$

and

$$
T\left(r, f_{\imath}\right)=(1+o(1)) T(r) \quad(i=1,2,3) .
$$

By Lemma 5 and (10), (13), (14) we also obtain (11), which is impossible. Assume $c_{1}=0$, from (9), (12) we have

$$
f_{1}+\left(1-\frac{c_{2}}{c_{3}}\right) f_{2} \equiv 1
$$

and

$$
T\left(r, f_{\imath}\right)=(1+o(1)) T(r) \quad(i=1,2,3),
$$

giving a contradiction as before.

Suppose that $f_{2} \equiv c(\neq 0)$. If $c \neq 1$, from (9) we have

$$
f_{1}+f_{3}=1-c
$$

and

$$
T\left(r, f_{\imath}\right)=(1+o(1)) T(r) \quad(i=1,2,3) .
$$

By Lemma 5 and (10), (14), (15) we obtain (11), which is impossible. Therefore $c=1$, that is, $f_{2} \equiv 1$.

Suppose that $f_{3} \equiv c(\neq 0)$. In a similar manner we get $f_{3} \equiv 1$.

This completes the proof of Lemma 7 . 
LEMMA 8. If, in addition to the assumptions of Theorem $2, f^{(n)} \equiv g^{(n)}$, then $f \equiv g$.

Proof. Suppose that $f \not \equiv g$. From $f^{(n)} \equiv g^{(n)}$, we have

$$
f(z)=g(z)+p(z),
$$

where $p(z)(\not \equiv 0)$ is a polynomial of degree at most $n-1$.

From $\delta(0, f)>0$ we know that $f$ is a transcendental entire function. Thus we get

and

$$
T(r, p)=o(T(r, f))
$$

$$
T(r, g)=(1+o(1)) T(r, f) .
$$

By the second fundamental theorem (see, [2, Theorem 2.5]), we have

$$
\begin{aligned}
T(r, f) & <N\left(r, \frac{1}{f}\right)+N\left(r, \frac{1}{f-p}\right)+S(r, f) \\
& =N\left(r, \frac{1}{f}\right)+N\left(r, \frac{1}{g}\right)+S(r, f) \\
& =2 N\left(r, \frac{1}{f}\right)+S(r, f) \\
& \leqq 2(1-\delta(0, f)) T(r, f)+S(r, f)
\end{aligned}
$$

Since

$$
2(1-\delta(0, f))<1
$$

so (16) is a contradiction. Hence $f \equiv g$.

\section{Proof of Theorem 2}

From $f^{(n)}=1 \rightleftarrows g^{(n)}=1$, we have

$$
f^{(n)}-1=e^{\alpha}\left(g^{(n)}-1\right),
$$

where $\alpha$ is a entire function.

Let $f_{1}=f^{(n)}, f_{2}=e^{\alpha}, f_{3}=-e^{\alpha} g^{(n)}$. From (17) we have

$$
\sum_{i=1}^{3} f_{i} \equiv 1
$$

and

$$
\sum_{i=1}^{3} N\left(r, \frac{1}{f_{i}}\right)=N\left(r, \frac{1}{f^{(n)}}\right)+N\left(r, \frac{1}{g^{(n)}}\right) .
$$

By Lemma 2 and Lemma 4 we have 


$$
N\left(r, \frac{1}{f^{(n)}}\right) \leqq T\left(r, f^{(n)}\right)-T(r, f)+N\left(r, \frac{1}{f}\right)+S\left(r, f^{(n)}\right)
$$

By Lemma 3 and Lemma 4 we have

$$
\begin{aligned}
N\left(r, \frac{1}{g^{(n)}}\right) & \leqq N\left(r, \frac{1}{g}\right)+S(r, g) \\
& =N\left(r, \frac{1}{f}\right)+S\left(r, f^{(n)}\right) .
\end{aligned}
$$

From (18), (19), (20) we obtain

$$
\begin{aligned}
\sum_{i=1}^{3} N\left(r, \frac{1}{f_{i}}\right) & \leqq T\left(r, f^{(n)}\right)-T(r, f)+2 N\left(r, \frac{1}{f}\right)+S\left(r, f^{(n)}\right) \\
& \leqq T\left(r, f^{(n)}\right)-T(r, f)+2(1-\delta(0, f)) T(r, f)+S\left(r, f^{(n)}\right) \\
& =T\left(r, f^{(n)}\right)-(2 \delta(0, f)-1) T(r, f)+S\left(r, f^{(n)}\right)
\end{aligned}
$$

By Lemma 1 and Lemma 4 we have

$$
T\left(r, f^{(n)}\right) \leqq T(r, f)+S\left(r, f^{(n)}\right) .
$$

Noting $2 \delta(0, f)-1>0$, from (21), (22), we get

$$
\begin{aligned}
\sum_{i=1}^{3} N\left(r, \frac{1}{f_{i}}\right) & \leqq T\left(r, f^{(n)}\right)-(2 \delta(0, f)-1) T\left(r, f^{(n)}\right)+S\left(r, f^{(n)}\right) \\
& =2(1-\delta(0, f)+o(1)) T\left(r, f^{(n)}\right) \\
& \leqq(\lambda+o(1)) T(r) \quad(r \notin E),
\end{aligned}
$$

where $\lambda=2(1-\delta(0, f))<1$. By Lemma 7 , we have $f_{2} \equiv 1$ or $f_{3} \equiv 1$.

If $f_{2} \equiv 1$, from (17) we have $f^{(n)} \equiv g^{(n)}$. By Lemma 8, we get $f \equiv g$. If $f_{3} \equiv 1$, from (17) we have $g^{(n)}=-e^{-\alpha}, f^{(n)}=-e^{\alpha}$, and hence $f^{(n)} \cdot g^{(n)} \equiv 1$. This completes the proof of Theorem 2 .

\section{REFERENCES}

[1] M. OzawA, Unicity theorems for entire functions, J. d'Analyse Math. 30 (1976), 411-420.

[2] W.K. Hayman, Meromorphic functions, Oxford. 1964.

[3] H. UEdA, Unicity theorems for meromorphic or entire functions II, Kodai Math. J. 6 (1983), 26-36.

[4] C.C. YANG, On two entire functions which together with their first derivatives have the same zeros, J. Math. Anal. Appl., 56 (1976), 1-6. 
[5] R. Nevanlinna, Le théorème de Picard-Borel et la théorie des fonctions méromorphes, Paris, Gauthier-Villars, 1929.

[6] F. Gross, Factorization of meromorphic functions, U.S. Govt. Printing Office Publication, Washington, D.C., 1972.

Department of Mathematics

SHANDONG UNIVERSITY

Jinan, Shandong, 250100

P. R. OF CHINA 